Portland State University

PDXScholar

Engineering and Technology Management

Faculty Publications and Presentations

\title{
Contingent Requirements for Artifical Intelligent Systems Development
}

\author{
Gary Langford \\ Portland State University, gary.langford@pdx.edu \\ Herman Migliore \\ Portland State University
}

Follow this and additional works at: https://pdxscholar.library.pdx.edu/etm_fac

Part of the Artificial Intelligence and Robotics Commons

Let us know how access to this document benefits you.

\section{Citation Details}

G. O. Langford and H. J. Migliore, "Contingent Requirements for Artifical Intelligent Systems Development," 2019 Portland International Conference on Management of Engineering and Technology (PICMET), Portland, OR, USA, 2019, pp. 1-9.1

This Article is brought to you for free and open access. It has been accepted for inclusion in Engineering and Technology Management Faculty Publications and Presentations by an authorized administrator of PDXScholar. Please contact us if we can make this document more accessible: pdxscholar@pdx.edu. 


\title{
Contingent Requirements for Artifical Intelligent Systems Development
}

\author{
Gary O. Langford, Herman J. Migliore \\ Engineering \& Technology Management, Portland State University, Portland, Oregon, USA
}

\begin{abstract}
A substantial portion of project failures are due to poorly defined requirements before enough is known about pragmatic end-item product capability, technology maturity, or development strategy. Process models either start with requirements or are weakly structured to elicit and derive actual stakeholder needs and to establish incontrovertible requirements. Existing process models are used acceptably for systems but are wholly inadequate for system and system of systems requirements that involve interactions with humans at a personal level. Problems with products and services are notable when artificial intelligent systems are put into use. Rather than establishing a technology baseline then working up requirements to advance and then use technology, a set of contingent requirements are posed to be promoted and advanced through a vetting process. From the technology chosen, a requirement is methodically planned to devise a capability, characteristic, or condition as part of the first phase of that vetting. This paper introduces a new form of requirements, termed contingent requirements. A contingent requirement stipulates the conditions for it to be modified according to the way things in fact are (or will be). From scenarios developed systematically by applying the Rand-Stanford method, technology dependencies are postulated, analyzed, and evaluated. The aim for introducing contingent requirements into the lifecycle engineering of Artificial Intelligent Systems (AIS) recognizes that technology should be considered within domains of influences, context(s), and consequences when put into use. Linear, incremental process models do not test for unforeseen consequences. Intelligent systems built on contingent requirements explore those unforeseen consequences by formally test the Intelligent Systems in various environments to explore the risks of interactions with individuals and in communities.
\end{abstract}

\section{INTRODUCTION}

Process models guide workflow by identifying and completing schedule and unscheduled work deemed necessary as measured at various milestones established by the key project stakeholders [1]. It is the goal of modeling processes to formulate a set of relations within the confines of a causal domain to answer questions, solve problems, resolve conflicts, and to explore ideas. For modeling a system of systems development, such as for an Artificial Intelligent Systems (AIS), there are four causal domains that incorporate the whole of product development. Each domain is uniquely distinguished as necessary and by the integration and interoperability of the work products from the four domains, made sufficient as the whole through the process model constructs used to consider when formulating requirements. These four questions of thinking are (a) which requirements are necessary and sufficient to capture the intended modeling objectives for systemic activities, processes, and mechanisms, (b) what problems may arise from omitting requirements that encapsulate the systemic nature of systems or systems of systems, (c) why are the conflicts in requirements between processes of the parts of constituent systems hampering the activities of the system of systems (the whole), and (d) how are the deficiencies in requirements from not building on systems modeling invalidating or limiting the validity of the development process model. Moreover, requirements for modeling systems of systems need validation to determine their fitness for use. In brief, the premise of the traditional process model is that stakeholders often determine the basic requirements from which additional requirements are derived. The typical recourse for poor requirements is to engage stakeholders, model the implications of those requirements, and prototype to confirm or note changes. Contingent requirements offer the pathway to determine the set of requirements before committing to a system of systems design that is fraught with tentacles of failure.

\section{APPROACH AND METHOD}

The central theme of systems theory identifies common patterns and principles that cross all types of objects that have similar properties. The similarities include observations, assumptions, definitions, and postulates applicable in scale from atoms to living things to galaxies. The codification into a general systems theory has been the focus since the 1950's. The systems approach has been attached to similarities in mathematics, living things, social organizations, business, processes, physical things, computers, and an ever-expanding array of all else. The result has been to dilute the meaning and relevance of the term systems, such that now virtually anything can and has been seemingly justified as a system.

The intent of this paper is to reinvigorate and impose a rather strict set of conditions by which systems can be identified and characterized with the same previous sense of similarities, albeit with a more rigorous explanation of the conditions that systems must satisfy. The approach here is to first, reflect on abstractions of the fundamental aspects of systems that have been noted by researchers in the past; and second, to expand on those particular similarities so that mechanisms can be identified, behavioral explanations can be verified, and predictions can be made to determine actionable means of improving the systemic nature of systems.

The result of an extensive examination of abstracted aggregations of various physical objects is explore the plethora of existing concepts from an entirely different perspective. That perspective is to abstract the behaviors 
indicative of the fundamental nature of all systems. Here, we observe that all systems to be metastable, have interactions with objects other than within their boundaries, to support their self-sustainment and be responsive to out-of-boundary interactions, and finally to produce non-reciprocal emergents.

In brief, the approach is to think systems and apply systems reasoning in a manner to survive and thrive. Thinking systems is different than systems reasoning. Thinking systems is focused on the ontological aspects of thinking within an integrative framework; whereas, systems reasoning is about explaining behaviors (i.e., mechanisms) in terms of a model of systems. That systems model satisfies the four conditions of systemsness (discussed in Section V).

\section{ARtificial Intelligent Systems (AIS)}

Artificial Intelligent Systems (AIS) are particularly vulnerable to mistaken, erroneous, false, imprecise, and missed requirements. An AIS development involves a higher level of complexity than a systems development or a not a system (notasystem) product or service. A notasystem is a set of physical objects interacting in such a way to keep being what it is and doing what it does by not being significantly affected by interactions from outside its boundaries [2]. Notasystems are stable without the feedback inherent in dynamically stable systems and systems of systems. A rock (notasystem) has no action - does nothing and cannot do anything without being acted upon by something else. In contrast, a system is interactive with external objects by initiating interactions and responding to outputs from other objects.

Our focus is on avoiding problems when initial requirements have either not yet been formulated or their formulation is lacking in some aspect(s). Here, we distinguish between aggregations of physical objects that are not systems, i.e., not-a-system (notasystem) and those aggregations of physical objects that satisfy the conditions of systems.

\section{Process Models}

Many excellent products and services have been produced by applying engineering process models for notasystems; and systems engineering process models for systems. However, neither an engineering process model nor a systems engineering process model will suffice for building AIS meant to interact with humans [3]. The impediment for these process models is that both the engineering and the systems engineering models rely wholly on a fairly robust set of requirements as their starting point. Both types of process models start by laying out a set of requirements. The process models proceed through further elicitation of needs from which to derive requirements, then defining functions as part of design, conceptualizing a functional architecture, then building and testing smaller subfunctions, and finally integrating and verifying the work to those requirements.

\section{A SYSTEM}

A system is the result of integration to satisfy four conditions - the criteria of systemness. The systemic behaviors are that of Metastability, Internal agility, External adaptability, and Non-reciprocal (irreversible) emergence (MIEN) [2]-[6]. Those systemic ways and matters are uniquely indicative of systems, regardless of scale, size, or provenance - from atoms to molecules, living things to AIS, to solar bodies and galaxies. All systems have the same criteria for their existence.

\section{A SySTEM OF SySTEMS}

A system of system exists only because the constituent systems work together to create the emergent functional performances that are not expressible within any of its individual system. A system of systems enhances the functional performances of all constituent systems. For a system of systems, the lifecycle nature of its existence means that its integration and interoperability drive the emergent capabilities [3]. Lifecycles have stages that progress from a beginning to an ending. Some emergent properties, traits, and attributes cannot exist outside of the system of systems. Each constituent system must also satisfy the criteria for being a system; and in addition, the aggregation of systems must not harm or prevent a system from retaining its systemness while participating within a system of systems [2-6]. And, any or all of the constituent systems shall not be harmed or prevented from being a system during the process of leaving the system of systems, from which point onward they are individually the systems they were prior to becoming part of the system of systems.

\section{LIFECYCLE}

The realities of a lifecycle can be thought of as the temporal interpretation of integration, whereas the events of integration during the lifecycle are interpretable in terms of advancing through the stages of lifecycle according to changes in the physical objects and processes associated with the activities and events of the system. Thinking in terms of event is typical of systems thinking for engineering products and service due to the fundamental nature of how these deliverables are realized, sustained, and matured. At the beginning of the lifecycle of a system of systems, constituent systems exist and start the process of becoming part of an integrated whole - the system of systems. Integration proceeds as physical object interact with each other, i.e., physical integration through interactions; and then advances to exchanging Energy, Matter, Material wealth, and Information (EMMI) to works toward a defined degree of interoperability. The degree of interoperability depends on the amount of control that the constituent systems will permit through the exchange of EMMI. There are suggestions that an integration process model [7]-[10] would be distinguishably different than a lifecycle process model for a notasystem or a system. It is through that lifecycle view of a system of systems, e.g., for AIS, that we realize the need for contingent requirements. Different than when objects and their interactions with EMMI 
are delineated, organized, and prioritized to lay out the blueprints for the task of building and testing, and integrating and testing, the system of systems is the emergent from two or more systems joining together by integration and interoperability. That emergence confounds the integration of two systems [3].

The constituent systems of a system of systems must retain their systemic nature before, during, and after becoming part of the system of systems. The systems of systems functionalities are emergent functions that either did not exist within the constituent systems and emerged from the integrated agglomeration of constituent systems or existed in one or more of the constituent systems and extended their functionalities to other constituent systems. In either case, the emergent function might exhibit change in functional performance or quality. In artificial systems of systems, the emergent is typically a change in performance or quality. In natural constituent systems, the emergent is typically a new function that is not expressed in any of the constituent systems. Therefore, systems of systems' emergence is related to the lifecycle of a particular system of systems. As the phase of the lifecycle changes so do the emergent properties, traits, and attributes change for the system of systems.

\section{VIII.CONTINGENT REQUIREMENTS}

The few existing process models used in technology management generally work with an established or technology development baseline, then work up requirements to advance and use technology when the product or service is released to customers and users. That aim is confounded by how that chosen technology is implemented and interacts with humans (in the case of AIS). From the technology perspective, a requirement is methodically planned to devise a capability, characteristic, or condition. This paper introduces a new form of posing requirements as contingent on the outcomes of the psychological consequences of AIS in various applications. A contingent requirement stipulates the conditions for it to be modified according to, (a) the way things in fact are (or will be), (b) by dependencies on other circumstances, or (c) by unacceptable consequences. The aim of introducing contingent requirements into the lifecycle engineering of Intelligent Systems recognizes that technology should be considered within domains of influences, context(s), and consequences when put into use. Linear, incremental process models do not test for unforeseen consequences. Intelligent systems built on contingent requirements explore those unforeseen consequences by formally testing the Intelligent Systems in various environments to explore the risks of interactions with individuals and in communities.

If a requirement is satisfied (and a need is met), the operation of the objects and process within the final product or service are said to be within the operational parameters of the system. If, however, a requirement is contingent on operability in a prescribed environment after the product or service is delivered, then the notion of a requirement that shall be incorporated in a product or service leaves wanting two conditions: necessity and contingency. Take for example, the need of the AIS to communicate with a human (intelligent system). There may be a stakeholder who wants to record that communication because the recording can provide feedback on how customers feel about interactions with AIS, to learn how customers express themselves with syntax and semantics when responding to questions and prompts, or to provide inputs to a voice-print database that can be used to identify and track interactions between that person with AIS and other devices or other individuals. That stakeholder "want" may be deemed sufficient to promote the idea to the level of a requirement, i.e., something that is necessary, sufficient, verifiable [6], and attainable within the scope of the project development work and thereafter deployment into operations with humans. Yet, the desirability of that stakeholder "want" may be at odds with legal issues, regulatory conditions, ethical considerations, and anti-social feelings. The perspectives of all stakeholders need to be considered, from which contingent requirements should be proposed and explored by survey, modeling, and applying a structured approach to generating a particularized theory that follows from logical assumptions and results in a qualitative understanding, of a specified set of conditions, including environment, context, demographics, circumstances, and possible scenarios, e.g., grounded theory [11].

Contingent requirements, in stark contrast to requirements, need to be investigated thoroughly, especially to ensure they are suitable for use, i.e., validated. Following Rudolf Carnap [12], the contingent requirement is logically true for conditions A but not for conditions B, logically false for conditions $\mathrm{C}$, implies conditions $\mathrm{D}$ when $\mathrm{A}$ is true, and is equivalent to conditions $\mathrm{E}$ when $\mathrm{A}$ is true. Consider a set of communications between AIS and a human where both parties agree tacitly first and then explicitly (without coercion, including by denying access to information requested by the human) to not record their interactions for the encounter. The communications indicate that the human desires information without providing data requested by AIS. The functions of 'recognize face', 'recognize voice', 'recognize syntax', 'recognize semantics', 'recognize breath chemistry', 'recognize mannerisms' are already operative and recording. According to logic rule set, that statement regarding the human desire to interoperate with AIS without complying with the AIS request for permission 'to record' is then true if and only if the both human and the AIS can verify and trust that said actions taken by each other are as each interprets same. Otherwise, the contingency status of a wanted requirement remains and the system design and architecture need to consider ramifications of all actions and events [2].

All requirements shall be first contingent requirements with the properties equivalent to facts - facts that are supported by logical truths. But the facts do not need to have a plethora of necessity or sufficiency, merely enough to be justified as acceptable to the stakeholder of the transaction between the individual and the AIS. For each transaction, these stakeholders might be transitory as the exchange of 
communications between human and AIS progresses. The key interaction - that of the human and the AIS - is the central focus for determining the principle actions of exchange that drive the relations between AIS and human. All that each of these two systems enact during the causal-affair that is at the center of the human and AIS transaction, shall be captured in the design of the AIS, the attitudes and habits of the human, and the architecture that is facilitates the system of systems. The transactions are to be orchestrated through that architecture according to the agreed upon rule set of truths. Therefore, the contingent requirements must incorporate the rule-set, context, and transaction(s) of the causal-affair.

It is the intent that the system of systems sprouts the contexts for normative behaviors of the human and AIS. Contingent requirements identify a capability or condition that could be met to ensure all stakeholders' needs are satisfied, but do not stipulate in what manner the need is to be met. A contingent requirement is an empirical assertion, but reasonable for the stakeholders. For those needs requiring testing for function performance(s) and quality, specific solutions involving physical objects shall be identified. Verification is to both the then stated requirement as well as the contingency requirement(s). In essence, the purpose of contingent requirements is to postulate a causally wanted response to particular contingencies that particularize interactions between humans and AIS. These contingent traits of a presumed social transaction that will have a certain outcome is then informed with behaviors indicative of a very wide range of potential enactors - both human and AIS. Each behavior needs to be characterized as being plausible under a very wide range of circumstances, justifiable by the needs of individuals, acceptable by users of AIS information, subject to laws, and socially responsible for the level of interaction expected by individuals participating in the transactions.

Not all contingent requirements will be deemed acceptable by all stakeholders. As is the case for any set of requirements, the negotiation pits human expectations and needs against AIS and its business model. The mediation between stakeholders extracts acceptable requirements from contingent requirements. The focal point from which all stakeholders have the basis for their at-risk participation in the AIS-human interactions is exactly during those AIS-human interactions.

\section{A. Coupling}

During interactions between systems or systems of systems, there must be integration of physical objects through exchanges of EMMI, i.e., connection and a sufficiency of coupling and cohesion to facilitate interoperability of EMMI [2]. All objects that interact have a degree of coupling. The concept of loose coupling forms a principle in architecting that states, the looser the dependency (e.g., coupling) between partitioned elements, the greater autonomy and flexibility one partitioned element has to satisfy the requirements to accept output from a partitioned element and provide input to another partitioned element. A measure of coupling between two objects is equivalent to the degree of dependency between them. The looser the coupling, the greater the autonomy and flexibility in satisfying the requirements of interoperability with other partitioned elements. Loose coupling means there are perhaps many ways of providing the necessary boundary conditions to accept inputs and release outputs to other partitioned elements. There is less linking of something inside a partitioned element with that of another partitioned element. No coupling means that partitioned element may not connect with other partitioned elements and very loosely coupled partitioned elements may be quite difficult to maintain. There is a balance between loosely coupled partitioned elements and very loosely coupled partitioned elements. The most basic measure of coupling is the number of linkages between one partitioned element and another partitioned element. The frequency and type of coupling improves the cost of maintaining these linkages. For a system, loosely coupled means being able to produce the same result with different means; indicative of a lack of coordination; operating without rules or regulations; and highly connected objects with high latency of information or otherwise very slow feedback time. According to Karl Weick, the benefits of very loosely coupled partitioned elements are resilience in a rapidly changing context or environment; fosters adaptation to local factors; encourages novel solutions to specific problems; encourages self-determination; promotes creativity; and develops leaders [13].

\section{B. Cohesion}

All coupled objects have varying extent of cohesion. The objective of decomposing functions is to identify those functions that are loosely coupled and highly cohesive. Loose coupling means there is an input and an output between functions that satisfies an object's boundary conditions and is used by the object's input mechanisms to produce an acceptable outcome. High cohesion means that when input is received, the function carries out exactly the set of operations intended by the input mechanism(s) and as expected by observation of the output functional performance(s). Cohesion is collaboration with the output being expected based on the input. Therefore, subfunctions are separable and hence conceptually independent; and respond to inputs and outputs that indicates operational independence. In this manner of object to object interaction, an end-to-end functional thread can be constructed to result in an action that is measurable and repeatable. Meeting the two conditions of being measurable and repeatable ensures that the intended function can be evaluated, controlled, verified and validated.

Connectivity (interaction), coupling (interoperability), and cohesion (responsiveness to requests) are foundational to the development of systems and systems of systems.

\section{Integration and Interoperability}

Integration and interoperability act as the catalyst to create awareness, appreciation, and responsiveness to these AIS-human interactions. Each stakeholder responds or acts to support the participants in the interaction, i.e., the human, the human as an agent(s) for others, the people who depend 
directly and indirectly (to include consequences thrice removed) on the actions and deeds as a consequence of the human-AIS interaction(s). Figure 1 illustrates the transaction topology for Humans and AIS. Agency agreements, contracts, and sales and leasing arrangements abound for businesses and individuals working with the AIS. Data moves from HUMAN to AIS to servers external to AIS to Companies (Co.) that provide back-end service functions and Co. that pay for data in keeping with their business models [10].

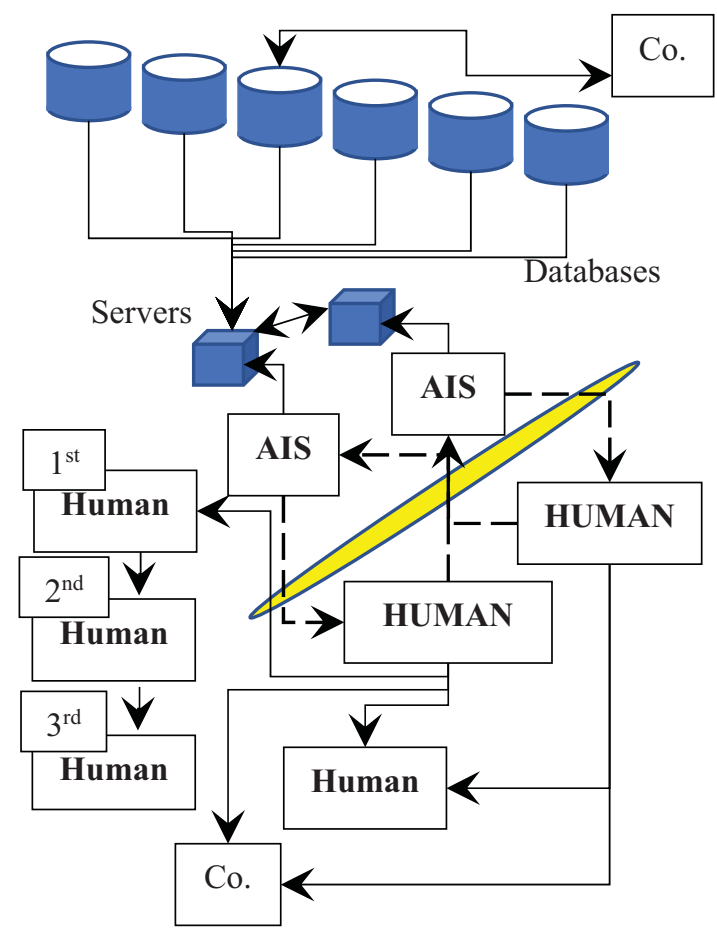

Fig. 1. AIS-HUMAN Transaction Topology

\section{Issues With AIS-Human Integration and Interoperability}

Several issues mediate the stakeholder perspectives about their needs and requirements. The manner and means of designing the AIS-HUMAN interface exposes the need for all stakeholders to insist on respect for the social graces that enwrap the interactions between AIS and Humans. Enthusiasm, intentions, reporting the experience, and an overall assessment results from those interactions. The contingency requirements should reflect each stakeholder's view of that AIS-Human interaction.

Additionally, the uncertainties of how the HUMAN user will react to the context and expressions of content within the AIS should be incorporated into the architecture that moves EMMI from object to object (through databases, inference engines, forecasting algorithms, and prognostication of future expectations. The priority afforded a particular AIS and Human interaction (in case resources are unavailable), the thoroughness of the response by the AIS, the timeliness of response to the needs of the human, and the setting of expectations by an AIS when interacting with humans will dictate the overall experience of the human participant. Here, we have not assumed a need for real-time operations that would place additional demands on providing just-in-time information. Contingent requirements may result in some stakeholders within the AIS domain system of systems being relegated to lesser priority than other stakeholders. There are simple, but perhaps ineffective solutions, such as pay for priority or fastest response to AIS to a query forms a queue base on response time. Simple to implement, yet perhaps ineffective based on the needs of the human.

\section{MINIMUM LosS}

Moreover, the overall utility of the transactions (i.e., the transaction's net worth and the interaction's net worth [2]) should be evaluated from the perspective of each stakeholder. A simple and perhaps effective solution to net sufficiency would be a determination of minimum loss for all stakeholders based on EMMI [14]. Figure 2 illustrates the application of a general loss function [2] to scheduling maintenance of aircraft where the causal significance of significant differences in costs of competing organizational is highlighted and the dependency of time away from one organization is interpretable as economic loss. The net result was an overall reduction in the cost of maintenance for both organizations when scheduling of shared resources was optimized for the minimum loss for the aggregation of organizations. There was no system of systems value for favoring one constituent organization over another consistent organization. The economic loss for the AIS system of systems is realized across all constituent systems. Attribution of those losses to achieve the minimum loss for all, needs to be negotiated congenially. The minimum loss is indicted by the dashed, blue vertical line.

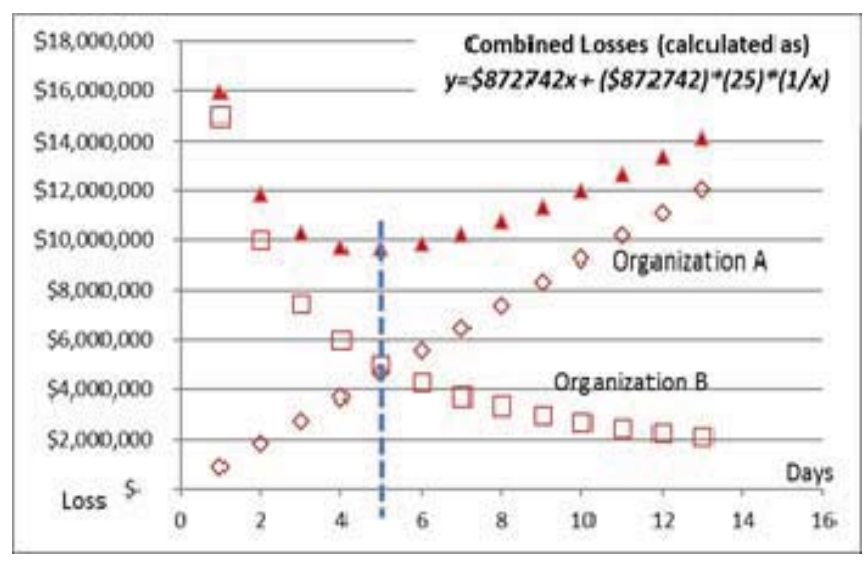

Fig. 2. Total Loss for Both Organizations Is Less Than Calculated Separately and Then Summed [14]

\section{A. Practicalities of Design for AIS}

Design for minimum loss across all interactions between AIS and Humans is a daunting task. Both extensional and intension logic must be considered as they are essential in discovering and accounting for all losses due to interaction. For every interaction, there is a loss - The Law of Action [2]. 
Every loss is the result of two objects interacting. Every interaction has extensional and intensional aspects [15].

\section{a) Extension}

Extension is determined by empiric methods. The name of something is an object, as are a thing, fact, proposition [2]. Retried knowledge from a computer is because of formal representations that are compliant with the logical and lexical semantics that ubiquitous imbue search and categories.

\section{b) Intension}

In contrast, intensional logic is indicative of most human reasoning. Intensional intelligence is built by comprehension rather than by all instances of empirical ideas and concept categories. Both intensional and extensional intelligence are necessary to provide a complete intelligent thought process.

\section{c) Measures of Success}

Monitoring the human acceptance of intensional logic (that capable of qualitatively describing based on internal content of a concept without empirical analysis or reasoning by lists of named ideas) [13] in AIS activities will be key drivers in establishing the normative views of the stakeholders resulting from negotiations and acceptance of the inherent results of tradeoffs in the final set of requirements. Here, the measure of success is the totality of human behaviors observed in surveys, social media, complaints, popular support for expanding AIS, and rate of increase and numbers of participants. Technology adoption curve(s) for AIS should be compared with data from radio, TV, cell phone, and Internet activities.

\section{Assessment OF NeEDS}

It is the interactions between AIS and Humans that dictate the contingent requirements. It is the nature of interaction, typified by the exchange of EMMI between two physical objects that mechanisms are created and enacted. Stakeholders have needs that often can be solved by skillfully employing mechanisms - the enabler of all things physical and behavioral. It is possible to achieve an interaction of a certain type and specificity at a lower level of operation that when combined with another interaction, results in an emergent behavior not observable at the lower level of interaction. Needs must be carefully examined to allow only acceptable behaviors. Examining the stakeholder needs using a systems model-based approach, we extrapolate several methodical analyses to expose functional requirements (we term requirements) through a precising formulation of useful emergents, i.e., functions. Note, model-based systems engineering approach (MBSE) is premised on set theory. The Systems Model-Based Thinking (SMBT) used here is based on the mereology of objects and processes, and proper parts.

\section{Fundamentals of Action in a System or System of SYSTEMS}

Systems and systems of systems produce an economy of action in excess what any part can do by itself. Physical boundaries can pass EMMI by mechanisms, if the boundary conditions allow. Functional boundaries, at the action due to the interface of contact between two objects proceeding therefrom to the last interaction with all other objects. Functional boundaries extend to the last interaction in time and space with all other objects. Changing an object's mechanism(s) or physical structure may change the function and functional performances that result from interactions with all other objects. To occur, that interaction must satisfy the boundary conditions of every two objects that attempt to interact. Therefore, as the EMMI moves from the initial interaction to the farthest extent its interaction at great distances may or may not be diminished by that travel. For example, neutrinos after billions of miles of travel in space lose negligible amounts of energy and retain their immense potency when interacting with Earth. In contrast, light energy decreases as the inverse square of the distance travelled. At immense distances the light from a star that is reflected from a planetary atmosphere may be insufficient to be detected by optical telescopes in low-earth orbit or ground-located larger reflecting surfaces. However, by observing the "wobble" in the orbit of that start, we may detect the effects of the mass of revolving planet around that star. The function of 'to detect exo-planets' can be due to gravitational forces rather than reflected starlight - the former revealing interaction between orbiting objects of a sufficiency of mass, the latter not satisfying the boundary conditions for detection from our telescope sensors. Behavioral boundaries are describable as how a sentient being reacts to observing or not observing an object (that may or may not be expected) and the reaction to observing the function of an interaction or the lack of a function (with or without that interaction).

\section{A. Functions and Processes}

Function is expressed by change, i.e., action that is measurable (relative to a standard). The quantification of change (by mechanisms) is functional performance. However, process is NOT function and function is NOT process. Process and function are different. And, we treat them distinctly different, although they are related in the same sense that energy (function) is related to force (process). A process is a partially ordered set of activities in a structured order, carried out within limitations, subject to tradeoffs (i.e., constraints) to provide a result that is intended to satisfy a need [2]. A function is a quantifiable result with a measurable performance that is required for a need to be satisfied [2].

\section{B. Proper Parts}

Interactions distribute EMMI to all proper parts within the structure of interacting objects. A proper part is taken as a primitive relation wherefrom nothing substantive follows from it. In other words, a proper part of a system or system of systems has a firm basis in reality and so important, meaningful, or considerable it is subject to the wisdom of minimum loss. Further, a proper part of a system or system of systems is one which is not identifiable as the whole or a part, but rather as an aggregation of parts of parts. Said differently, and more formally, an aggregation of objects $P$ is an object such that everything which overlaps with it also overlaps with 
something in $P$ and vice versa. Figure 3 visualizes the mathematics of proper part.

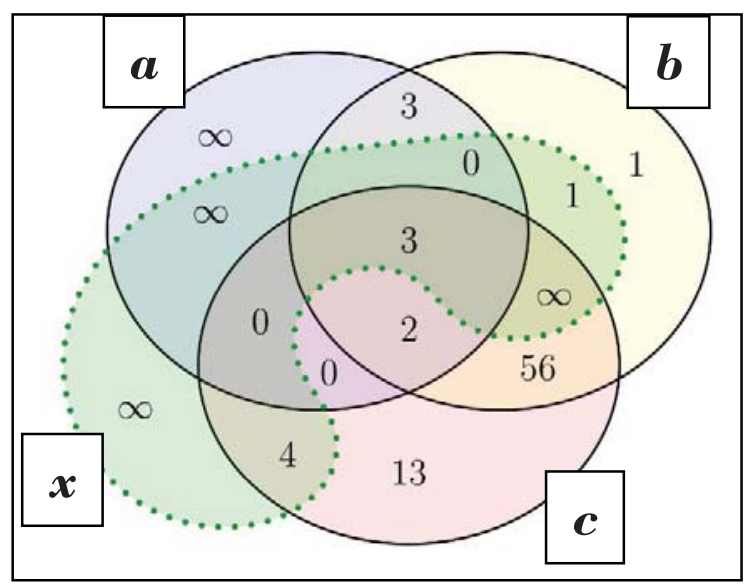

Fig. 3. Ingrediens of The Proper Parts of Object $P$

\section{Contingent Requirements to Requirements}

Joining and participating in a system of systems is meant to enhance a constituent's functional performances. When a system's requirement is subject to change due to joining or participating in a system of systems, the loss needs to be considered. Therefore, system requirement (before the system of system) may become a contingent requirement that must be reexamined as one to be considered, expressed, and then retained (within the context of the system), but may be part of a system of systems tradeoff, shunted to another system, or relinquished altogether.

Once a contingent requirement is determined to be an acceptable system of systems requirement, that requirement may be allocated to any one or many of the constituent systems as a Proper Part (note the visualization of cross system participation in Proper Parts). System of systems requirements are therefore testable (within the system of systems context, verifiable to the system of systems specification which reference the system of systems requirements, and stipulated as a system of systems functional requirement that benefit all constituent systems (assuming Proper connectivity, coupling, and cohesion).

A key determinant for a system of systems requirement is its determination as a Proper Part of the system of systems. Scenarios [13] describe events or activities that synopsize a sequence of intentions or responses to events so that an accounting for a plausible future can be methodically posed and vetted against various methods of that pose other plausible futures. Scenarios are the futurity of events. The task is to use different methods to expose various paths to the future. Scenarios help discover missing or influential stakeholders (i.e., find additional stakeholders, problems, needs, and requirements); uncover possible sequences of events that are causal; and to develop strategies to explore consequences and uncertainties that follow from anticipated interactions between AIS and humans. Scenarios can be used to ascertain the desirable types of integrations and interoperabilities needed by all constituent systems within a system of systems. Based on patterns of planned behaviors the contingent requirements can be examined and validated. Validation of future uses of a product or service (being problematic in general) requires people other than today's users to posit those future uses [2]. "Today's users are steeped in today's product and service, and oftentimes find difficulties extracting themselves from today's thinking" [2]. Great care must be taken to discern future uses.

\section{Scenario Development}

A Bayesian interpretation of scenario development leads to the defining of the order of developments, the optimum or causal starting time(s) for development, and the duration of a proposed effort. The result is presented as probabilistic measures of stakeholder beliefs that certain patterns are possible (subject to all caveats one can imagine). The probability of an event occurring in a particular sequence, at the designated starting time, and having the posed duration is dependent on the complexity of the events and the knowledge of the events [16]. Various scenarios can be scripted and evaluated.

Through the work of Rand Corporation and Stanford Research Institute, four techniques have been demonstrated to discern the patterns and behaviors that offer reasonable and accurate scenarios for futures work. The four are referred to as demonstration scenario [17]-[21], driving force [22]-[25], system change [25], and slice-of-time [26].

\section{a) Demonstration Scenario}

The demonstration scenario posits clearly outlined and reasonable sequences of decisive events along with decision points where alternative sequences are considered. To avoid what Wayne Boucher terms "genius" forecasting, we use a forward-looking sequence with backwards-looking validation, revise the decision points to be alternative decision points where a myriad of decisions is concatenated into each of the sequence steps. In other words, the demonstration is shown for each segment within the chain. When combined with one of the other four types of scenarios, this hybrid demonstration method was shown to be accurate and was validated on several projects for the U.S. Army. As modified the hybrid demonstration method is used by the authors for planning within a 25 -year horizon.

\section{b) Driving-Force Scenario}

The driving-force scenario depends on key trends at the time from which the planning begins. This type of scenario builds on historical data and as such is quite popular with commercial, financial, and government planners. The primary assumption is the driving forces remain constant. The impact of potential events is ignored when developing a baseline for the future. Consequently, the driving-force scenario is redone when a primary assumption changes. Thus, the driving-force scenario has a limited 6-month to 2-year horizon. Our experience with the driving-force scenario planner is reasonably accurate if the updates are completed every 6months.

\section{c) System-Change Scenario}

The system-change scenario is meant to enhance the demonstration scenario and the driving force scenario by taking into consideration a more system-like thinking. Unlike 
the typical enactments of the system-change scenario, we apply a Systems Model-Based Thinking (SMBT) to determining the range of possible interactions and their likely causal impacts [2]-[6], [14], [15]. The essence of SMBT is the recognition that a system and a system of system (with their corresponding contingent requirements) represent aggregations of physical objects and processes all of which are operative for the functions necessary to continue the existence of the self. The concept that wholes and parts are interactive and therefore monistic. Anything can therefore exist or not exist, or not be possible to exist. The Leśniewskian mereology of objects and processes form two distinct ontologies that when combined make up that which can exist and the rules by which that existence is possible. The consequence of the emergents from objects that interact is a set of functions and performances, mechanisms and ways of using same, behaviors and models that expose the possible causal events at each segment in the sequence that makes up the system change scenario. Though there is a significant learning curve to use SMBT, the result is a highly valuable approach, methods, and tools to advance longterm prognostications using the system-change scenario.

\section{d) Slice-of-Time Scenario}

The slice-of-time scenario poses a future and then either work backwards to present day or identify megatrends as John Naisbitt did from Stanford Research Institute [26]. The conception of megatrends improves both the demonstration and driving force scenarios. And, the megatrends help reduce the number of plausible outcomes from the system-change scenario. The combination of SMBT with slice-of-time scenario develop is wonderfully predictive - a strong recommendation from us.

\section{CONCLUSIONS}

With an effective approach to prognosticating the Proper mix of parts within systems and systems of systems, the development and use of AIS with humans will be simultaneously difficult and rewarding. At stake are juxtaposed AIS and humans trapped in a strained relation, where neither system cannot exist without the other. Contexts influence the effectiveness of requirements that have consequences in opposition. Contingent requirements allow stakeholders (e.g., developers, customers, and users) to both explore and exploit consequences. The choice to be made with contingent requirements is not to choose any one particular requirement, but rather to select and promote requirements that satisfy a need for short-term value that is consistent with the nexus of performance, cost, and schedule goals. In contrast, long-term goals often contribute to requirements that impose multi-variate tradeoffs that can reasonably be accomplished with a more mature consideration of requirements that have resulted in experience and feedback from operational use of the product or service. Contingent requirements are estimable for both short-term and long-term goals. At risk without contingent requirements is the development of improperly thought through AIS products and services that will suffer from poorly defined requirements. This paper identifies a key issue of requirements that must be addressed to achieve a more congenial future - a more exact formulation resulting from thinking systems and apply systems reasoning.

\section{REFERENCES}

[1] G. O. Langford, D. Burns, A. Keller, Paper 19R0279, "Domain Process Model Overcomes Limitations of Engineering Models for Developing Artificial Intelligent Systems," PICMET, 2019.

[2] G. O. Langford, Engineering Systems Integration: Theory, Metrics and Methods. Boca Raton, Florida, CRC Press/Taylor \& Francis. 2012.

[3] G. O. Langford, "System of Systems Process Model," Chapter 7, Engineering Emergence: A Modeling and Simulation Approach, L. Rainey and M. Jamshidi, Eds. Boca Raton, Florida: CRC Press/Taylor \& Francis. 2018.

[4] G. O. Langford and T. S-Y. Langford, "The making of a system of systems: Ontology reveals the true nature of emergence," in Conf. Proc. 2017 12th Annual System of Systems Engineering Conference IEEE Int.

[5] G. O. Langford, "Toward a General Theory of Systems Integration: Research in the Context of Systems Engineering," Ph.D. dissertation, Defence and Systems Institute, School of Electrical and Information Engineering, University of South Australia, Mawson Lakes, Australia, 2013.

[6] G. O. Langford. "Verification of requirements: system of systems theory, framework, formalisms, validity. 27th Annual INCOSE International Symposium," IS 2017, International Council on Systems Engineering, Adelaide, Australia, July 15-20.

[7] Project Management Institute, A Guide to the Project Management Body of Knowledge. Newtown Square, Pennsylvania, 2000.

[8] H.R. Booher, "Introduction: Human Systems Integration, in H.R. Booher (ed.), Handbook of Human Systems Integration," Chapter1, 130, New York, John Wiley and Sons, 2003.

[9] R. Jain, A., Chandrasekaran, E. Ozgur, "A Systems Integration Framework for Process Analysis and Improvement, Systems Engineering, 13(2), pp. 274-289, 2010.

[10] A. P. Tvaryanas, A Discourse on Human Systems Integration. PhD dissertation, Monterey: Naval Postgraduate School, 642pp, 2010.

[11] B. G. Glaser, and A. L. Strauss, The Discovery of Grounded Theory: Strategies for Qualitative Research. New York: Aldine Publishing Company, 1967.

[12] R. Carnap, Meaning and Necessity: A Study in Semantics and Modal Logic," The University of Chicago Press, Chicago, IL, Section 2, 1947.

[13] K. Weick, "Educational organizations as loosely coupled systems," Administrative Science Quarterly, 21, 1-9 (part), 1976.

[14] G. O. Langford, "Maintenance Scheduling Using Systems Engineering Integration," 26th Annual International Council on Systems Engineering (INCOSE), IS 2016, Edinburgh, Scotland, 18-21 July, 2016.

[15] G. O. Langford, J. R. Carpenter, L. Beaulieu, I. Watkins, B. Marsh, C. Chase, and T. Heidorn, "Intelligent Systems in Space," Paper 19R0266, Portland International Center for Management of Engineering and Technology (PICMET), Technology Management in the World of Intelligent Systems, In Press, 25- 29 August 2019.

[16] G. D. Peterson, G. Cumming, and S. R. Carpenter, Scenario planning: A tool for conservation in an uncertain world. Conservation Biology 17(2): 358-366, 2003.

[17] K. L. Poh, Utility-Based Categorization Engineering-Economic Systems. PhD thesis, Stanford: Stanford University, 238pp, 1993.

[18] H. Kahn, and A. Wiener, The Year 2000. New York: Macmillan, 1967.

[19] D. E. Sage and R. B. Chobot, The scenario as an approach to studying the future. In S. P. Henckley and J. R. Yates (eds.), Futurism in Education: Methodologies (pp. 161-178). Berkeley, CA: McCutchen, 1974.

[20] J. P. Martino, Technological Forecasting for Decision-Making (2nd ed.). New York: North-Holland, 1983.

[21] I. A. Wilson, Scenarios. In J. Fowles (ed.), Handbook for Futures Research (pp. 225-247). Westport, CT: Greenwood Press, 1978. 
[22] W. I. Boucher and J. L. Morrison, Alternative Recruiting Environments for the U.S. Army. Alexandria, VA: Army Research Institute, 1989.

[23] D. L. Goldfarb and W. W. Huss, Building scenarios for an electric utility. Long-Range Planning 21: 79-85, 1988

[24] W. C. Ashley and L. Hall, Nonextrapolative strategy. In J. S. Mendell (ed.), Nonextrapolative Methods in Business Forecasting (pp. 61-76) Westport, CT: Quorum Books 1985.
[25] T. F. Mandel, Futures scenarios and their use in corporate strategy. In K. J. Albert (ed.), The Strategic Management Handbook (pp. 10-21). New York: McGraw-Hill, 1983.

[26] Hawken, J. Ogilvy, and P. Schwartz, Seven Tomorrows. New York: Banlam, 1982. 